Submitted to Ceramics International, December 2015 (Revised February 2016)

\title{
Formulation and Micro-Extrusion of High Concentration Graphene Slurries
}

\author{
Ling Li, Shaik Mohamed Imran Ayub, and Leon L. Shaw* \\ Department of Mechanical, Materials, and Aerospace Engineering \\ Illinois Institute of Technology, Chicago, IL 60616
}

\begin{abstract}
In this study we have investigated the feasibility of preparing high concentration graphene slurries and their rheological properties as a function of various parameters including the sonicating time, solid loading, surfactant concentration, different types of graphene, aqueous and non-aqueous solvents. The feasibility of micro-extruding slurries with high graphene loading has also been studied. We demonstrate that slurries with graphene loading at $13 \mathrm{wt}$ \% (equivalent to $6.3 \mathrm{vol} . \%$ ) can be prepared to have pseudoplastic (shear thinning) properties. The 6.3 vol.\% graphene is the highest graphene loading ever reported in the open literature. An unusual phenomenon of increasing graphene loading leading to reduced viscosity after graphene loading has reached a critical value has been observed for the first time. This unusual phenomenon is attributed to the forced alignment of graphene flakes during shearing when graphene loading is high enough and has been utilized to prepare slurries with high graphene loading and pseudoplastic properties. Micro-extrusion of these high concentration graphene slurries reveals the capability of the extrudate to maintain its shape right after extrusion of the slurries. Thus, it is expected that these high concentration graphene slurries can be used to fabricate three-dimensional objects through layer-by-layer fabrication methods with high fabrication rates in the near future.
\end{abstract}

Keywords: Graphene; Slurry; Micro-extrusion; Pseudoplastic.

* Corresponding author: Leon Shaw, 1shaw2@iit.edu 


\section{Introduction}

Graphene with high specific surface area, electrical conductivity, thermal conductivity and mechanical strength has attracted tremendous attention in recent years [1-5]. Its theoretical specific surface area is $\sim 2630 \mathrm{~m}^{2} / \mathrm{g}$ and its electrical conductivity is measured to be $\sim 64 \mathrm{mS} / \mathrm{cm}$ which is approximately $\sim 60$ times more than that of single-walled carbon nanotubes [2-4]. The unique combination of its ultrahigh specific surface area and outstanding electrical conductivity along with its chemical stability makes graphene a strong contender for many energy related applications such as solar cells, batteries, electrochemical capacitors (ECs), and hydrogen storage [2-5].

However, in some applications such as ECs the difficulty in processing graphene nanosheets (GNs) is a concern. GNs have a high tendency to restack themselves during all phases of GN preparation and electrode fabrication processes, leaving behind inter-graphene pore sizes that are not sufficient to be accessible by the electrolyte and for the formation of electrical double layer (EDL) charges [6-9]. Goh and Pumera [10] have shown that it is not always beneficial to exfoliate graphitic structures to single layer graphene, multilayer (> 10 layers) graphene nanoribbons with cross sections of $100 \times 100 \mathrm{~nm}$ provide a larger capacitance $(15.6 \mathrm{~F} / \mathrm{g})$ than fewlayer (3-9 layers) graphene nanoribbons $(14.9 \mathrm{~F} / \mathrm{g})$ and a far greater capacitance than single layer GNs $(10.9 \mathrm{~F} / \mathrm{g})$ with the same cross section, likely caused by graphene layers laying flat on top of one another blocking/concealing the edge plane. One way to obtain higher effective surface area is to prevent graphene sheets from agglomeration [11]. Along this line, Guardia, et al. [12] have reported that non-ionic surfactants can exfoliate and disperse pristine graphene from graphite in water at significant concentrations (up to $1 \mathrm{mg} / \mathrm{mL}$ ). Lee, et al. [13] have shown that easily soluble expanded graphite (ESEG) can be sufficiently dispersed in an aqueous solution using an ordinary surfactant and various organic solvents, such as N-methyl-2-pyrrolidone (NMP), dimethylacetamide (DMAC), diethylene glycol (DEG), toluene, dimethylformamide (DMF), sodium dodecylbenzenesulfonate (SDBS), dichloromethane (DCM), and sodium dodecyle sulphate (SDS) [13]. In spite of the advances made so far, the typical graphene loading for stable slurries is below $1 \mathrm{wt} \%$ [12-16].

Screen printing and inkjet printing techniques are widely used for the fabrication of graphene based EC electrodes because of low cost. The concentration of graphene in inks is often limited by many requirements such as small particles sizes, suitable viscosity and printability. 
Therefore, only very low graphene concentrations (up to $63 \mathrm{~g} / \mathrm{L}$ ) [17-21] can be prepared for inks. In order to achieve high loading of graphene dispersions with suitable viscosity properties, surfactants for aqueous systems or toxic solvents for non-aqueous systems are often applied [22]. In contrast to screen printing and inkjet printing, Nathan-Walleser, et al. [23] have reported a micro-extrusion method to process ECs using binder-free thermally reduced graphene oxide (TRGO) in both aqueous and non-aqueous electrolyte systems. The solid loading is up to $15 \mathrm{~g} / \mathrm{L}$, and their ECs display a volumetric capacitance of $10 \mathrm{~F} / \mathrm{cm}^{3}$ in $1 \mathrm{M} \mathrm{KOH}$ electrolyte and $8.5 \mathrm{~F} / \mathrm{cm}^{3}$ in the electrolyte with $1 \mathrm{M}$ tetraethylammonium tetrafluoroborate dissolved in acetonitrile (TEABF $4-A C N)$. The energy density of the TRGO-TEABF $4-A C N$ system is $4.43 \mathrm{mWh} / \mathrm{cm}^{3}$ and the power capability is up to $42.74 \mathrm{~kW} / \mathrm{cm}^{3}$ [23].

In this study, we have investigated the formulation and extrudability of graphene-based slurries in order to establish slurries with high graphene loading and suitable for micro-extrusion to fabricate ECs with superior electrochemical performance in the near future. Slurries with high graphene loading can result in high production rates and low shrinkage after micro-extrusion. Effects of various parameters including the sonicating time, solid loading, surfactant concentration, different types of graphene, aqueous and non-aqueous solvents have all been investigated. All of these parameters are found to have significant effects on the rheological properties of graphene-based slurries. Furthermore, it is found that slurries with 12.5 wt.\% graphene loading can be prepared with proper pseudoplastic properties. These slurries have been micro-extruded successfully to form 3-dimensional shapes layer by layer fully controlled by a computer. Thus, this work lays a solid foundation for fabricating 3D graphene-based objects and devices through layer-by-layer methods in the near future.

\section{Experimental}

\subsection{Preparation of graphene slurries}

In all the slurries studied, the solid is composed $90 \mathrm{wt} . \%$ graphene plus $10 \mathrm{wt} . \%$ carbon black (CB), denoted as graphene/CB hereafter. CB is added to minimize the problem of graphene restacking themselves. It is assumed that uniform dispersion and mixing of graphene and $\mathrm{CB}$ during the slurry preparation stage can be inherited after slurry micro-extrusion. As schematically depicted in Figure 1, uniform presence of CB between GNs can physically prevent restacking of 
GNs. In order to achieve high solid loading slurries with pseudoplastic properties, we have investigated two systems, one being non-aqueous slurries and the other aqueous slurries. Two types of graphene are investigated as well. Graphene I is from Angstron Materials (Dayton, $\mathrm{OH}$ ), whereas graphene II is provided by Skysping Nanomaterials (Houston, TX). The specification from the vendor for Graphene I is 3- to 4-layer graphene with a thickness of 1 to $1.2 \mathrm{~nm}$ and the $\mathrm{x}$-y dimension less than $10 \mu \mathrm{m}$, whereas Graphene II is specified as graphene nanopowder with a thickness of 6 to $8 \mathrm{~nm}$ and average $\mathrm{x}-\mathrm{y}$ dimensions of $15 \mu \mathrm{m}$. The CB used is CNERGY Super C65 from Timcal Graphite \& Carbon (Westlake, OH). The CB is specified to have a specific surface area of $62 \mathrm{~m}^{2} / \mathrm{g}$ and most of its particles are less than $20 \mu \mathrm{m}$ in diameter.

Graphene and CB were suspended in NMP (Alfa Aesar, Ward Hill, MA) to prepare nonaqueous slurries or in deionized water with SDS surfactant (Fisher Scientific, Pittsburgh, PA) to study aqueous slurries. NMP is widely studied as an organic solvent for graphene slurries because it can disperse graphene well $[13,24]$, whereas SDS is an anionic surfactant which can help disperse graphene well in aqueous solvents [13]. Aqueous slurries were prepared by dispersing different weight percentages of graphene and CB (in a 9-to-1 weight ratio, termed as graphene/CB hereafter) in deionized water with various surfactant concentrations. In order to get uniform slurries, we employed a tipped sonicator (FB-120, Fisher Scientific) to disperse graphene/CB into deionized water or NMP. In order to make slurries micro-extrudable, all the slurries prepared were sonicated for 1 hour at $50 \%$ power outputs, followed by 1-hour ball milling with stainless steel balls of 0.25 inch in diameter using a SPEX mill to break up agglomerates and reduce graphene particle size (to be discussed more later). The ball milled slurries were then evacuated to remove the air trapped in the slurries before micro-extrusion.

To ensure the reproducibility of the data, we prepared three samples for each condition and tested the viscosities of these three samples as soon as sonication was completed (i.e., within 2 min after sonication). The data presented in this paper was the average of the three samples. Furthermore, it was found that the viscosity variation among the three samples was very small and in many cases the variation was smaller than the data points shown in the figures of this paper. To illustrate this point, some figures are presented with the average of the three samples along with the error bars showing the standard deviation. 


\subsection{Three-dimensional micro-extrusion}

The micro-extrusion of graphene slurries was conducted using a custom-designed multimaterial micro-extrusion machine which consists of an XYZ gantry system with a rectilinear motion control platform coupled with an extrusion system and a control interface to monitor and control the extrusion process by a computer. The rectilinear XYZ gantry system permits the microextrusion nozzle to move in $3 \mathrm{D}$ space with a position resolution at $\pm 11 \mu \mathrm{m}$. The extrusion system is made up of two gas tight syringes pushed by two separate high torque linear actuating cylinders. The moving speed and direction of the nozzle and the extrusion rate of the slurry are independently controlled through a multi-axial CNC control system linked to a computer. In this study, two different nozzles with openings of $210 \mu \mathrm{m}$ and $840 \mu \mathrm{m}$ in diameter were used to evaluate the micro-extrusion behavior of the selected slurries.

\subsection{Characterization of graphene and graphene slurries}

The surface area analysis of graphene was carried out using the Brunauer, Emmett and Teller (BET) method, employing a NOVA 2200e surface area analyzer from Quantachrome Instruments (Boynton Beach, FL). The morphology of graphene was examined through scanning electron microscopy (SEM), using a Hitachi S4700-II (Schaumburg, IL) at Argonne National Laboratory (ANL) or a JEOL 6335 (Tokyo, Japan) at the University of Connecticut. All SEM samples were gold-palladium sputter coated before imaging to avoid any possible charging effect. Raman spectra were obtained with an Ar ion laser operated at $514.5 \mathrm{~nm}$ using an inVia Raman spectrometer from Renishaw Inc. (Hoffman Estates, IL). The rheological properties of graphene slurries were characterized through viscosity measurements using a LVDV-II + PRO viscometer (Brookfield, MA).

\section{Results and discussion}

\subsection{Characteristics of Graphene}

Surface area measurements reveal that the specific surface area (SSA) of graphene II is 180 $\mathrm{m}^{2} / \mathrm{g}$, whereas the SSA of graphene I is $598 \mathrm{~m}^{2} / \mathrm{g}$ that is about three times the SSA of graphene II. These measurements are consistent with those reported by the vendors $\left(400 \mathrm{~m}^{2} / \mathrm{g}-800 \mathrm{~m}^{2} / \mathrm{g}\right.$ for graphene I and $120-150 \mathrm{~m}^{2} / \mathrm{g}$ for graphene II). Note that the SSAs of both graphenes are lower 
than the theoretic value of single layer graphene $\left(2675 \mathrm{~m}^{2} / \mathrm{g}\right)$ [5]. Based on the BET surface area analysis, one can estimate that graphene I is 4-layer graphene if no re-stacking occurs during sample preparation for the BET surface area analysis. Similarly, graphene II can be estimated from the BET surface area analysis to have about 14 layers.

Figure 2 shows the SEM images of graphene I and II. Note that most of graphene I particles have $x-y$ dimensions less than $10 \mu \mathrm{m}$ (Figure 2A), while graphene II contains many particles with $\mathrm{x}-\mathrm{y}$ dimensions in the range of 10 to $30 \mu \mathrm{m}$ (Figure 2B). Both measurements are consistent with the vendors' specifications. Thus, graphene I not only has a thinner thickness, but also has smaller $\mathrm{x}-\mathrm{y}$ dimensions than graphene II. The smaller particle size and larger SSA of graphene I are expected to result in more difficulty in dispersing graphene I in slurries than graphene II. In contrast, to micro-extrude graphene II slurries, larger nozzles have to be used because of its larger particle size. To solve this problem, some graphene II slurries after sonication have been subjected to 1-hour ball milling using a SPEX mill to break up agglomerates and reduce particle sizes. As shown in Figure 3, the graphene particle size after 1-hour ball milling becomes more uniform and smaller comparing to those before ball milling. Based on the image $\mathrm{J}$ analysis, it is found that 1hour ball milling has reduced $90 \%$ graphene particles to sizes between 1 to $10 \mu \mathrm{m}$ for their $\mathrm{x}-\mathrm{y}$ dimensions. This reduction greatly enhances extrudability of the slurries.

Figure 4 compares Raman spectra of graphene I and II. Note that both graphenes exhibit three peaks - the D peak at around $1350 \mathrm{~cm}^{-1}$, the $\mathrm{G}$ peak at around $1580 \mathrm{~cm}^{-1}$, and the $2 \mathrm{D}$ band at around $2700 \mathrm{~cm}^{-1}$. Graphene I has an additional D+D' band at $\sim 2961 \mathrm{~cm}^{-1}$, whereas graphene II does not have this band. It is well known that the $\mathrm{G}$ band arises from the in-plane vibration of $\mathrm{sp}^{2}$ carbon atoms of graphene, whereas the $\mathrm{D}$ band arises because of the disorder induced in $\mathrm{sp}^{2}-$ bonded carbon [25]. Further, the peak intensity ratio between the D and G-bands usually provides a useful index for comparing the degree of crystallinity of carbon materials, that is, the smaller the ratio of $\mathrm{I}_{\mathrm{D}} / \mathrm{I}_{\mathrm{G}}$, the higher the degree of ordering in the carbon material [26]. Since the $\mathrm{I}_{\mathrm{D}} / \mathrm{I}_{\mathrm{G}}$ ratio of graphene I is substantially larger than that of graphene II, it can be concluded that graphene I contains more defects than graphene II. This conclusion is also supported by the fact that graphene I has a strong D+D' peak, whereas the D+D' peak is nearly absent for graphene II. Previous studies $[27,28]$ have shown that graphene with significant defects would exhibit additional disorder related peaks, D' and D+D' at $\sim 1620$ and $\sim 2950 \mathrm{~cm}^{-1}$, originating from the intravalley double resonance and a combination mode, respectively. The intensity of the D' peak is typically very small [28]. 
Further, the position of the D' peak is overlapped with that of the broad G peak in the present study, and thus its presence is hard to be judged without conducting peak deconvolution. Nevertheless, the prominent presence of the $\mathrm{D}+\mathrm{D}$ ' peak at $\sim 2961 \mathrm{~cm}^{-1}$ exhibited by graphene I has unambiguously revealed that graphene I has more defects than graphene II, in good accordance with the high $\mathrm{I}_{\mathrm{D}} / \mathrm{I}_{\mathrm{G}}$ ratio of graphene $\mathrm{I}$.

Another interesting phenomenon of these Raman spectra is the position of the 2D band at $2695.5 \mathrm{~cm}^{-1}$ for graphene I, whereas it is at $2728 \mathrm{~cm}^{-1}$ for graphene II. A previous study [29] has found that the $2 \mathrm{D}$ band is located at $\sim 2690 \mathrm{~cm}^{-1}$ for single-layer graphene and up-shifts as the number of layers in graphene increases. When the number of layers increases to 5, the 2D band has shifted to $\sim 2728 \mathrm{~cm}^{-1}$ and becomes hardly distinguishable from that of bulk graphite [29]. Based on this prior study [29], we can conclude that graphene II has 5-layer graphitic structures or more since the position of its 2D band coincides with that of bulk graphite. Furthermore, graphene I has more than 1 layer but less than 5 layers since the position of its $2 \mathrm{D}$ band is at $2695.5 \mathrm{~cm}^{-1}$ (between 2690 and $2728 \mathrm{~cm}^{-1}$ ). It is interesting to note that the conclusion obtained from Raman data corroborates well with the estimation of the number of layers in graphene I and II derived from the BET surface area measurements.

\subsection{The Effects of Sonicating Time on Viscosity}

Non-aqueous graphene I/CB slurries using NMP as solvent with 2 wt.\% solid loading (i.e., $1.8 \mathrm{wt} . \%$ graphene I and $0.2 \mathrm{wt} . \% \mathrm{CB}$ ) were studied to determine the influence of the sonicating time. As shown in Figure 5, the viscosity of the slurry decreases with increasing the sonication time. We propose that sonicating promotes dispersion of graphene as well as wetting of graphene by NMP. As a result, longer sonicating time leads to more uniform dispersion of graphene and better wetting by NMP, which in turn results in lower viscosities. Based on these results, we have chosen to sonicate most of the slurries for 64 minutes before we carry out various characterizations. Thus, unless mentioned specifically, all slurries are sonicated for $64 \mathrm{~min}$ before characterization.

Note that all slurries in Figure 5 exhibit shear thinning properties, suggesting that dynamic agglomeration of graphene particles and breaking up of agglomerates are taking place during shear deformation. The higher the shear rate, the more effective in breaking up agglomerates and thus the lower viscosity. Longer sonicating times result in fewer agglomerates and thus lead to less shear thinning behavior as shown in Figure 5. This is consistent with the observation of lower 
viscosity with increasing the sonication time.

\subsection{The Effects of Solid Loading on Viscosity}

Non-aqueous and aqueous slurries have both been investigated for the influence of solid loading on viscosity. For the NMP solvent system, slurries with 3 wt.\%, 4 wt.\%, 5 wt.\%, 6 wt.\%, $7 \mathrm{wt} . \%$ and $8 \mathrm{wt} . \%$ of graphene II/CB are studied. The results are presented in two figures (Figures 6 and 7) to facilitate reading. As shown in Figure 6, the viscosity increases with solid loading in the range of 3 to $5 \mathrm{wt} . \%$ graphene II/CB. Such a phenomenon is known for slurries containing spherical particles and well documented in the literature [30-32]. However, it is interesting to note that when solid loading is increased to above $5 \mathrm{wt} . \%$, the viscosity decreases with increasing solid loading (Figure 7). This unusual phenomenon indicates the presence of a critical solid loading above which increasing solid loading results in a decreased viscosity. Such a phenomenon has never been reported before. However, to our surprise, such unusual phenomenon is also present in aqueous slurries, as described below.

For aqueous slurries with $0.015 \mathrm{M} \mathrm{SDS}$, it is found that the slurry with 4 wt.\% graphene $\mathrm{II} / \mathrm{CB}$ has a very low viscosity in the entire shear rate range (around $2 \mathrm{cp}$ only). However, the slurry with 5 wt.\% graphene II/CB exhibits much higher viscosities (Figure 8), suggesting that the viscosity of aqueous slurries is very sensitive to graphene solid loading. An even more interesting phenomenon is that the slurry with $6 \mathrm{wt}$ \% graphene II/CB has lower viscosities than slurries with lower solid loading (e.g., with 5 wt.\% solid loading as shown in Figure 8). This result indicates that $5 \mathrm{wt} . \%$ graphene II/CB is the critical solid loading for slurries with $0.015 \mathrm{M}$ SDS. Above this critical loading the viscosity decreases with increasing solid loading.

For aqueous slurries with $0.03 \mathrm{M}$ SDS, slurry with 8 wt.\% graphene II/CB has higher viscosity than slurry with 9 wt.\% graphene II/CB (Figure 9), indicating that the critical solid loading is $8 \mathrm{wt} . \%$ for the slurries with $0.03 \mathrm{M}$ SDS. For aqueous slurries with $0.06 \mathrm{M}$ SDS, solid loading of $10 \mathrm{wt} . \%$ graphene II/CB leads to dilatant behavior, and the viscosity increases with solid loading until $12 \mathrm{wt} . \%$ graphene II/CB (Figure 10). Above $12 \mathrm{wt} \% \%$ graphene II/CB the viscosity of the slurry decreases with increasing solid loading, indicating that $12 \mathrm{wt} \%$ solid loading is the

critical value above which the unusual phenomenon - the viscosity decreases with increasing solid loading - takes place.

Based on the results described above, it can be concluded that loading of graphene II/CB 
in either aqueous or non-aqueous solvent will lead to the unusual phenomenon discovered in this study, i.e., the viscosity of slurries decreases with increasing solid loading after solid loading reaches a critical value. Such a phenomenon has never been observed before for any materials. We propose that such an unusual phenomenon is related to the flake morphology of graphene. When solid loading is low, graphene flakes have random orientation and exhibit the usual behavior of the increased viscosity with solid loading [30-32]. However, when solid loading is high, graphene flakes are forced to align parallel during flow, which leads to reduced viscosity.

The mechanism proposed above is also consistent with another experimental observation made in this study, that is, the solid loading range for exhibiting the unusual phenomenon of the reduced viscosity with increasing solid loading is small for aqueous slurries. For example, for slurries with $0.015 \mathrm{M} \mathrm{SDS}$, the viscosity of the slurry decreases when solid loading increases from 5 wt.\% to 6 wt.\% (Figure 8). However, when solid loading is increased to 7 wt.\%, the slurry becomes so thick that its viscosity cannot be determined using the LVDV-II+PRO viscometer, indicating that the viscosity value is higher than $12,000,000 \mathrm{cp}$ (the upper limit of the viscometer). Similarly, for slurries with $0.03 \mathrm{M}$ SDS, the viscosity of the slurry decreases when solid loading increases from 8 to $9 \mathrm{wt} . \%$ (Figure 9). However, when solid loading is increased to $10 \mathrm{wt} . \%$, the viscosity exceeds the upper limit of the viscometer. Similar situation occurs to slurries with $0.06 \mathrm{M}$ SDS as well, i.e., the solid loading range for exhibiting the unusual phenomenon of the reduced viscosity with increasing solid loading is small. The small solid loading range within which the unusual phenomenon occurs may be attributed to very strong interactions among graphene particles when solid loading is much higher than the critical value, even with the alignment of graphene flakes forced by high solid loading.

To further explore the critical concentration phenomenon, we have investigated the sonicating time effect. It is found that the critical concentration of the aqueous graphene II/CB slurry with $0.06 \mathrm{M}$ SDS is $12.5 \mathrm{wt} . \%$ when the sonicating time is 64 minutes, but becomes $5 \mathrm{wt} . \%$ when the sonicating time is reduced to 16 minutes. Thus, the critical concentration is not only a function of the surfactant concentration, but also a function of the sonicating time. Figure 11 compares the rheological behavior of two aqueous slurries with the critical concentrations of graphene II/CB loading after 64-min and 16-min sonicating times. First of all, it should be noted that the viscosity variation among the three samples under each condition is very small because the error bars are, in many cases, smaller than the data points in the figure. Further, it is interesting 
to note that the slurry with the 64-min sonicating time has a very high viscosity at the low shear rate region $(<101 / \mathrm{s})$, but decreases to approach the viscosity of the slurry with the 16-min sonicating time at the high shear rate region $(>201 / \mathrm{s})$. We hypothesize that this phenomenon is likely related to exfoliation of multi-layer graphene (graphene II) which could take place during sonication in the presence of the SDS surfactant. In fact, it has been reported that exfoliation of graphene under sonication in the presence of surfactants (such as SDBS) is extremely successful $[33,34]$. Thus, we hypothesize that a longer sonicating time results in the formation of more graphene flakes which in turn lead to more interaction among graphene flakes and thus higher viscosities when the shear rate is small. However, at high shear rates most of graphene flakes are aligned and thus viscosities reduce significantly. Further, the viscosity difference between the slurries with a larger number of graphene flakes (64-min sonicated slurry) and a smaller number of graphene flakes (16-min sonicated slurry) becomes small after the alignment of graphene flakes (i.e., at the high shear rate region).

In this study, we have utilized this unusual critical concentration phenomenon to prepare slurries with high graphene loading and at the same time possessing pseudoplastic properties. Such slurries will increase the fabrication rate because of the high solid loading and provide the desired properties for micro-extrusion to achieve low extrusion pressure while offering the capability to maintain extrudate's shape right after micro-extrusion. The latter is critical in controlling the dimensions of 3D objects; otherwise, the extruded slurry will spread, leading to little control of the $3 \mathrm{D}$ object dimensions.

\subsection{The Effects of Surfactant Concentration on Viscosity}

In this study SDS is added to aqueous slurries as a surfactant. It is found that SDS is very effective in reducing the viscosity and increasing solid loading. As shown in Figure 12, increasing the SDS concentration leads to reduced viscosities and increased solid loading simultaneously. Specifically, for slurries with 0.015M SDS, the highest solid loading achievable is $6 \mathrm{wt} . \%$ (with a viscosity of $\sim 1400 \mathrm{cp}$ at low shear rates) beyond which the viscosity of the slurry is too high to be measurable using our viscometer. For slurries with $0.03 \mathrm{M}$ SDS, the highest solid loading achievable increases to $9 \mathrm{wt} . \%$, while the viscosity reduces to $780 \mathrm{cp}$ at low shear rates. For slurries with $0.06 \mathrm{M}$ SDS, the highest solid loading achievable increases further to $13 \mathrm{wt} . \%$ with the viscosity decreasing further to $\sim 200 \mathrm{cp}$. 
The phenomena discussed above are likely related to the coverage of SDS on the surface of graphene. As the SDS concentration increases, more surface area of graphene is covered by SDS and thus graphene is better dispersed, which reduces the interaction between graphene particles, thereby allowing the increase in solid loading while reducing the viscosity. In this study, we have not yet observed the upper limit of the SDS concentration above which the viscosity does not decrease any more, suggesting that further increase in the SDS concentration may increase solid loading further. Such a possibility will be investigated in the near future.

\subsection{The Effect of the Surface Area of Graphene on Viscosity}

As mentioned above, the specific surface area of graphene $\mathrm{I}\left(598 \mathrm{~m}^{2} / \mathrm{g}\right)$ is three times the specific surface area of graphene II $\left(180 \mathrm{~m}^{2} / \mathrm{g}\right)$. It is found that the dispersibility of graphene is strongly affected by its specific surface area. For example, the highest loading of graphene I is 2 wt.\% in the non-aqueous solvent (NMP), while graphene II could reach $8 \mathrm{wt} \%$ in the same solvent. Such a phenomenon is very similar to the often-observed increase in viscosity when particle size is reduced [31]. For a given weight, an increase in the specific surface area is equivalent to a decrease in particle size and/or an increase in the number of particles. In this study, the higher SSA of graphene I is due to an increase in the number of graphene as well as a decrease in graphene size (both thickness and $x-y$ dimensions, see discussion in Section 3.1). Therefore, for slurries with a given weight of graphene, one can expect higher viscosities for graphene I slurries than graphene II slurries because of more interactions among a larger number of graphene I particles. The higher degree of defects in graphene I, as revealed from the Raman analysis, may also increase the interaction between individual particles during dispersion, making graphene I more difficult to disperse than graphene II.

\subsection{Extrudability of Graphene Slurries}

Some studies have been conducted to find out whether high solid loading slurries are suitable for micro-extrusion. It is found that $12.5 \mathrm{wt} . \%$ graphene II/CB slurries with $0.06 \mathrm{M}$ SDS after 1-hour ball milling and removal of the air trapped in the slurries could be successfully extruded using a $18 \mathrm{G}$ (inner diameter: $840 \mu \mathrm{m}$ ) nozzle. Note that $12.5 \mathrm{wt} . \%$ graphene/CB in aqueous solvent is equivalent to $6 \mathrm{vol} \%$ graphene/CB if the densities of graphene and $\mathrm{CB}$ are assumed to be $2.2 \mathrm{~g} / \mathrm{cm}^{3}$. Because of their pseudoplastic properties, these slurries can be micro- 
extruded with low pressure while maintaining their shapes and dimensions as soon as the extrudate has been pushed out of the nozzle. However, slurries with solid loading lower than $12.5 \mathrm{wt} \%$ graphene II/CB have poor extrudability because these slurries are too dilute and cannot maintain extrudate's shape right after micro-extrusion. Instead, the extrudate spreads out after microextrusion, leading to little control on the shape and dimensions of the extrudate. Figure 13 shows IIT letters produced from 2 passes of micro-extrusion, while Figure 14 displays a single wall fabricated by stacking 7 passes of micro-extrusion. Clearly, 3D shapes can be produced from micro-extrusion of high graphene-loaded slurries with proper pseudoplastic properties.

It should be mentioned that the present micro-extrusion studies have also revealed several undesirable phenomena, including (a) phase separation, (b) clogging during micro-extrusion, and (c) air trapped in the slurries. All these phenomena have made micro-extrusion of graphene slurries imperfect. It is found that clogging and air trapped in the syringe made the extrusion discontinuous which led to the printed lines with different widths, as shown in Figure 13. The air trapped in the slurry is generated during the slurry preparation stage as well as upon loading of the slurry into the reservoir. However, the trapped air can be minimized by carefully performing vacuum degasing during slurry preparation and loading of the slurry into the reservoir under a sub-vacuum glovebox. Phase separation happens near the end of micro-extrusion. Clogging and phase separation are due to large graphene sizes $(\sim 15 \mu \mathrm{m}$ in the $\mathrm{x}-\mathrm{y}$ dimension) in comparison to the nozzle size $(840 \mu \mathrm{m})$. The ratio of the nozzle size to the graphene size is about 56 times, which is lower than ideal. Larger nozzle sizes or smaller graphene sizes should result in complete removal of clogging and phase separation.

\section{Conclusions}

Slurry formulations with two types of graphene (graphene I and graphene II) dispersed in non-aqueous and aqueous solvents have been investigated. The following interesting phenomena have been observed. (i) Increasing the sonicating time decreases the viscosity of graphene I slurries. This is attributed to the promotion of dispersion of graphene I and wetting of graphene I by the solvent through sonicating. (ii) Increasing the SDS concentration decreases the viscosity of graphene slurries, indicating that SDS is an effective dispersant for graphene in water. (iii) Graphene I with fewer layers is more difficult to disperse than graphene II with more layers, and 
thus more graphene can be loaded into slurries for graphene II than graphene I. This is due to the larger specific surface area of graphene I and thus stronger interactions between graphene particles when compared with graphene II. (iv) An unusual phenomenon is observed for the solid loading effect. The viscosity of slurries increases with graphene loading when solid loading is low. However, the viscosity of slurries decreases with increasing graphene loading when solid loading is above a critical value. This "critical concentration" phenomenon has been interpreted by a change from random orientation of graphene to parallel alignment during flow when solid loading is high, and utilized to prepare slurries with high solid loading and pseudoplastic properties. (v) Slurries with 12.5 wt.\% graphene II/CB exhibit proper pseudoplastic properties and have been micro-extruded successfully. Slurries with $12.5 \mathrm{wt} \%$ graphene II/CB loading (equivalent to 6 vol.\% loading) have the highest graphene loading among any extrudable or printable slurries ever reported in the literature. The breakthrough made in this study is expected to drastically improve the production rate for fabricating $3 \mathrm{D}$ objects made of graphene in the near future.

Acknowledgements - The financial support from US National Science Foundation with grant number CBET 1252924 is greatly appreciated.

\section{References}

1. A.K. Geim and K.S. Novoselov, The rise of graphene, Nature Mater. 6 (2007) 183-191.

2. D.A.C. Brownson, D.K. Kampouris, and C.E. Banks, An overview of graphene in energy production and storage applications, J. Power Sources 196 (2011) 4873-4885.

3. C. Liu, S. Alwarappan, Z. Chen, X. Kong, and C.Z. Li, Membraneless enzymatic biofuel cells based on graphene nanosheets, Biosens. Bioelectron., 25 (2010) 1829-1833.

4. X. Wang, L. Zhi, and K. Mullen, Transparent, conductive graphene electrodes for dyesensitized solar cells, Nano Lett. 8 (2008) 323-327.

5. P.M. Hallam and C.E. Banks, Quantifying the electron transfer sites of graphene, Electrochem Commun. 13 (2011) 8-11.

6. C. Liu, Z. Yu, D. Neff, A. Zhamu, and B.Z. Jang, Graphene-based supercapacitor with an ultrahigh energy density, Nano Lett. 10 (2010) 4863-4868. 
7. Y. Wang, Z. Shi, Y. Huang, Y. Ma, C. Wang, M. Chen, and Y. Chen, Supercapacitor devices based on graphene materials, J. Phys. Chem. C 113 (2009) 13103-13107.

8. M.D. Stoller, S. Park, Y. Zhu, J. An, and R.S. Ruoff, Graphene-based ultracapacitors, Nano Lett. 8 (2008) 3498-3502.

9. D. Yu and L. Dai, Self-assembled graphene/carbon nanotube hybrid films for supercapacitor, J. Phys. Chem. Lett. 1 (2010) 467-470.

10. M.S. Goh and M. Pumera, Multilayer graphene nanoribbons exhibit larger capacitance than their few-layer and single-layer graphene counterparts, Electrochem. Commun. 12 (2010) 1375-1377.

11. S.L. Candelaria, Y. Shao, W. Zhou, X. Li, J. Xiao, J. Zhang, Y. Wang, J. Liu, J. Li, and G. Cao, Nanostructured carbon for energy storage and conversion, Nano Energy 1 (2012) 195220.

12. L. Guardia, M.J. Fernandez-Merino, J.I. Paredes, P. Solis-Fernandez, S. Villar-Rodil, A. Martınez-Alonso, and J.M.D. Tascon, High-throughput production of pristine graphene in an aqueous dispersion assisted by non-ionic surfactants, Carbon 49 (2011) 1653-1662.

13. J.H. Lee, D.W. Shin, V.G. Makotchenko, A.S. Nazarov, V.E. Fedorov, J.H. Yoo, S.M. Yu, J.Y. Choi, J.M. Kim, and J.B. Yoo, The superior dispersion of easily soluble graphite, Small 6 (2010) 58-62.

14. D. Wang, D. Choi, J. Li, Z. Yang, Z. Nie, R. Kou, D. Hu, C.M. Wang, L.V. Saraf, J. Zhang, I.A. Aksay, and J. Liu, Self-assembled $\mathrm{TiO}_{2}$-graphene hybrid nanostructures for enhanced Li-ion insertion, ACS Nano 3 (2009) 907-914.

15. K. Zhang, L. Mao, L.L. Zhang, H.S.O. Chan, X.S. Zhao, and J. Wu, Surfactant-intercalated, chemically reduced graphene oxide for high performance supercapacitor electrodes, J. Mater Chem. 21 (2011) 7302-7307.

16. P. Chen, H. Chen, J. Qiu, and C. Zhou, Inkjet printing of single-walled carbon nanotube/ $\mathrm{RuO}_{2}$ nanowire supercapacitors on cloth fabrics and flexible substrates, Nano Res., 3 (2010) 594-603.

17. L.T. Le, M.H. Ervin, H. Qiu, B.E. Fuchs, and W.Y. Lee, Graphene supercapacitor electrodes fabricated by inkjet printing and thermal reduction of graphene oxide, Electrochem. Commun. 13 (2011) 355-358. 
18. J. Li, F. Ye, S. Vaziri, M. Muhammed, and M.C. Lemme, Efficient inkjet printing of graphene, Adv. Mater. 25 (2013) 3985-3992.

19. Y. Xu, I. Hennig, D. Freyberg, A.J. Strudwick, M.G. Schwab, T. Weitz, and K.C.P. Cha, Inkjet-printed energy storage device using graphene/polyaniline inks, J. Power Sources 248 (2014) 483-488.

20. E.B. Secor, P.L. Prabhumirashi, K. Puntambekar, M.L. Geier, and M.C. Hersam, Inkjet printing of high conductivity, flexible graphene patterns, J. Phys. Chem. Lett. 4 (2013) 13471351.

21. Umar Khan, Harshit Porwal, Arlene O’Neill, Khalid Nawaz, Peter May, and Jonathan N. Coleman, Solvent-Exfoliated Graphene at Extremely High Concentration, Langmuir 27 (2011) 9077-9082.

22. T. Hasan, F. Torrisi, Z. Sun, D. Popa, V. Nicolosi, G. Privitera, F. Bonaccorso, and A.C. Ferrari, Solution-phase exfoliation of graphite for ultrafast photonics, Phys. Status Solidi B 247 (2010) 2953-2957.

23. T. Nathan-Walleser, I.-M Lazar, M. Fabritius, F.J. Tölle, Q. Xia, B. Bruchmann, S.S. Venkataraman, M.G. Schwab, and R. Mülhaupt, 3D micro-extrusion of graphene-based active electrodes: towards high-rate AC line filtering performance electrochemical capacitors, Adv. Funct. Mater. 24 (2014) 4706-4716.

24. J. Xia, F. Chen, J. Li, and N. Tao, Measurement of the quantum capacitance of graphene, Nat. Nanotechnol. 4 (2009) 505-509.

25. M. Su, Z. Wang, H. Guo, X. Li, S. Huang, W. Xiao, and L. Gan, Enhancement of the cyclability of aSi/graphite@graphene composite as anode for lithium-ion batteries, Electrochimica Acta, 116 (2014) 230-236.

26. J. Liang, S. Chen, M. Xie, Y. Wang, X. Guo, X. Guo, and W. Ding, Expeditious fabrication of flower-like hierarchical mesoporous carbon superstructures as supercapacitor electrode materials, J. Mater. Chem. A 2 (2014) 16884-16891.

27. J. Hong, J. K. Park, E. J. Lee, D. Lee, D. S. Hwang, and S. Ryu, Origin of new broad Raman D and G peaks in annealed graphene, Sci. Rep. 3 (2013) 2700, DOI: 10.1038/srep02700.

28. D. C. Elias, R. R. Nair, T. M. G. Mohiuddin, S. V. Morozov, P. Blake, M. P. Halsall, A. C. Ferrari, D. W. Boukhvalov, M. I. Katsnelson, A. K. Geim, and K. S. Novoselov, Control of 
graphene's properties by reversible hydrogenation: evidence for graphene, Science 323 (2009) 610-613.

29. A. C. Ferrari, J. C. Meyer, V. Scardaci, C. Casiraghi, M. Lazzeri, F. Mauri, S. Piscanec, D. Jiang, K. S. Novoselov, S. Roth, and A. K. Geim, The Raman fingerprint of graphene, Phys. Rev. Lett. 97 (2006) 187401-187405.

30. D. J. Kim, H. Kim, and J. K. Lee, Dependence of the rheological behavior of electrostatically stabilized alumina slurries on $\mathrm{pH}$ and solid loading, J. Mater. Sci. 33 (1998) 2931-2935.

31. P. K. Senpati, D. Panda, and A. Parida, Predicting viscosity of limestone-water slurry, J. Minerals Mater. Characterization Eng. 8 (2009) 203-221.

32. N. Behabtu, J. R. Lomeda, M. J. Green, A. L. Higginbotham, A. Sinitskii, D. V. Kosynkin, D. Tsentalovich, A. N. G. Parra-Vasquez, J. Schmidt, E. Kesselman, Y. Cohen, Y. Talmon, J. M. Tour, and M. Pasquali, Spontaneous high-concentration dispersions and liquid crystals of graphene, Nature nanotechnol. 5 (2010) 406-411.

33. A. Ciesielski and P. Samori, Graphene via sonication assisted liquid-phase exfoliation, Chem. Soc. Rev. (2013) DOI: 10.1039/c3cs60217f.

34. M. Lotya, Y. Hernandez, P. J. King, R. J. Smith, V. Nicolosi, L. S. Karlsson, F. M. Blighe, S. De, Z. M. Wang, I. T. McGovern, G. S. Duesberg, and J. N. Coleman, Liquid phase production of graphene by exfoliation of graphite in surfactant/water solutions, J. Am. Chem. Soc. 131 (2009) 3611-3620. 


\section{Figure Caption}

Figure 1. Schematic of the function of uniform mixing carbon black with graphene to prevent graphene restacking themselves.

Figure 2. SEM images of graphene: (A) graphene I and (B) graphene II. Note that different magnifications are used.

Figure 3. SEM image of the aqueous slurry with 12.5 wt.\% graphene II/CB and 0.06M SDS after 1-hour ball milling.

Figure 4. Raman spectra of (a) graphene I and (b) graphene II with a 514nm excitation laser wavelength.

Figure 5. Viscosity as a function of the shear rate and sonicating time for non-aqueous slurries containing 2 wt. \% of graphene I and 0.2 wt.\% CB. Sonicating time: $8 \min (\bullet), 16 \min (\boldsymbol{\Delta}), 32 \min (\bullet)$, and $64 \min$ $(\diamond)$.

Figure 6. Viscosity as a function of the shear rate and solid loading for non-aqueous slurries. Solid loading

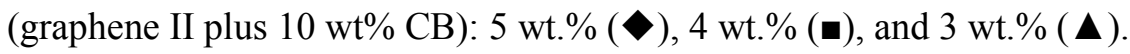

Figure 7. Viscosity as a function of the shear rate and solid loading for non-aqueous slurries. Solid loading

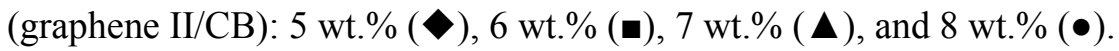

Figure 8. Viscosity as a function of the shear rate and solid loading for aqueous slurries with $0.015 \mathrm{M}$ SDS. Solid loading (graphene II/CB): 4 wt.\% (•) 5 wt.\% (•), and 6 wt.\% (

Figure 9. Viscosity as a function of the shear rate and solid loading for aqueous slurries with 0.03M SDS. Solid loading (graphene II/CB): 8 wt.\% (•) and 9 wt.\% (घ).

Figure 10. Viscosity as a function of the shear rate and solid loading for aqueous slurries with 0.06M SDS.

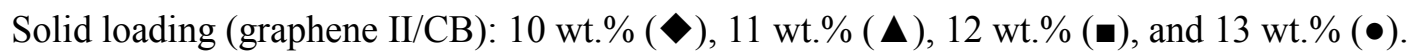

Figure 11. Viscosity as a function of the shear rate for aqueous slurries with $0.06 \mathrm{M}$ SDS but different sonicating times as indicated. Solid loading (graphene II/CB): 12.5 wt.\% for 64 -minute sonication $(\diamond)$ and 5 wt.\% for 16 - 
minute sonication $(\bullet)$. The error-bars in the figure represent the standard deviation and in many cases are smaller than the symbols shown in the figure, indicating the high reproducibility of the experiments.

Figure 12. Viscosity as a function of the shear rate and SDS concentration for aqueous slurries. The graphene II/CB loading and SDS concentration: 6 wt.\% solid loading/0.015M SDS ( $), 9$ wt.\% solid loading/0.03M SDS (•), and 13 wt.\% solid loading/0.06M SDS ( $\mathbf{\Delta})$.

Figure 13. IIT letters fabricated through micro-extrusion of the aqueous slurry with $12.5 \mathrm{wt} . \%$ graphene II/CB using an 18G nozzle.

Figure 14. A single wall fabricated by stacking multiple lines from micro-extrusion of the aqueous slurry with 12.5 wt.\% graphene II/CB using an $18 \mathrm{G}$ nozzle. 


\section{Figures}

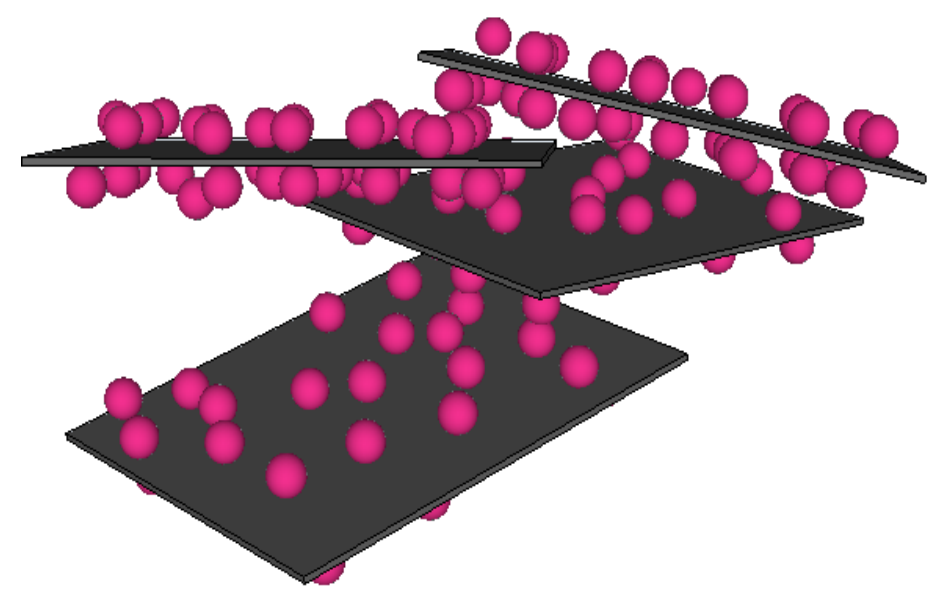

Figure 1. Schematic of the function of uniform mixing carbon black with graphene to prevent graphene restacking themselves.
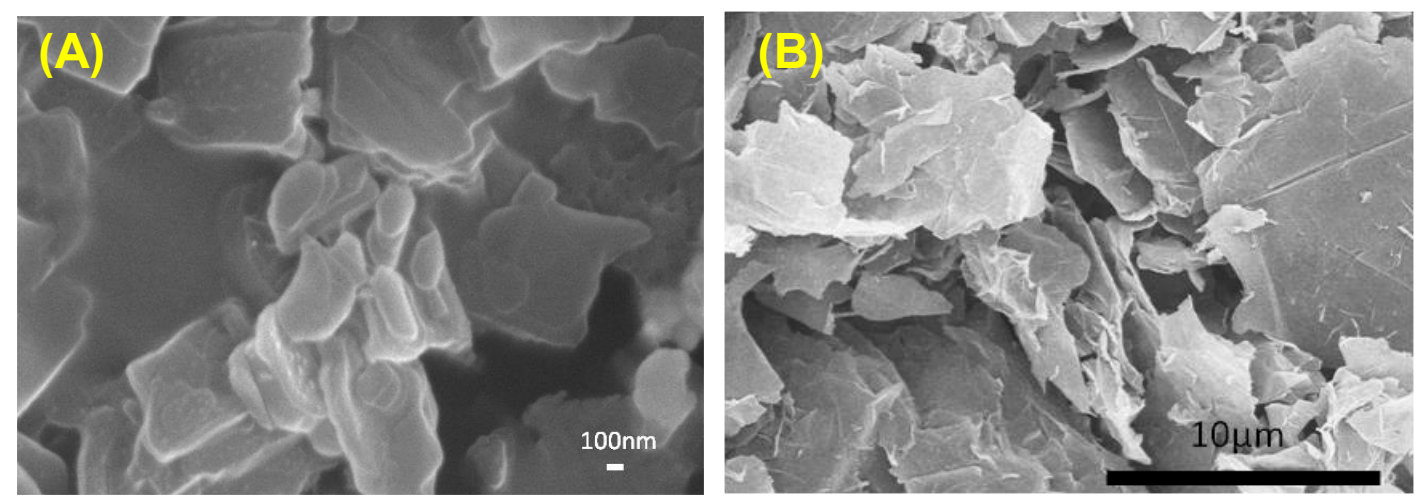

Figure 2. SEM images of graphene: (A) graphene I and (B) graphene II. Note that different magnifications are used. 


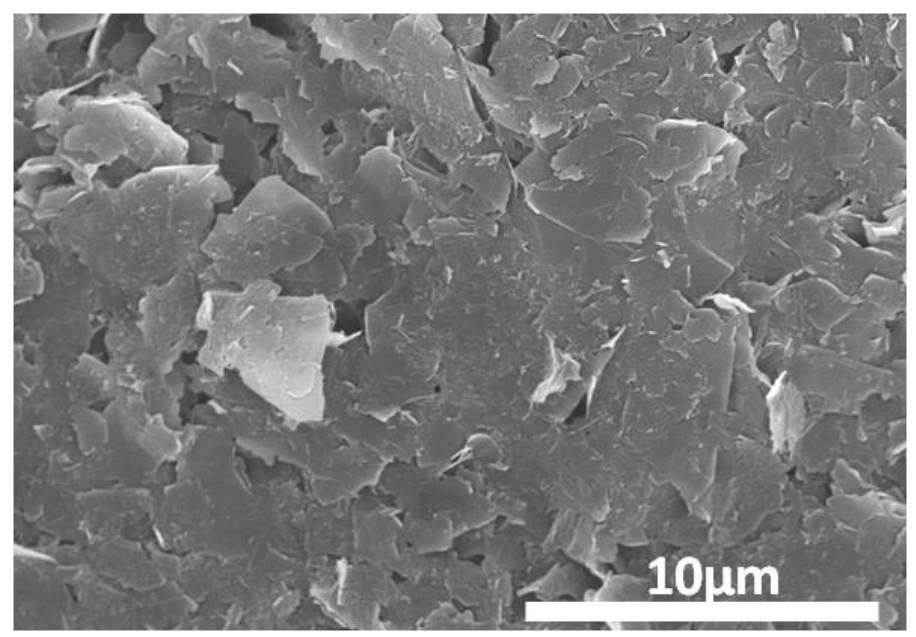

Figure 3. SEM image of the aqueous slurry with $12.5 \mathrm{wt} \%$ graphene II/CB and 0.06M SDS after 1-hour ball milling.
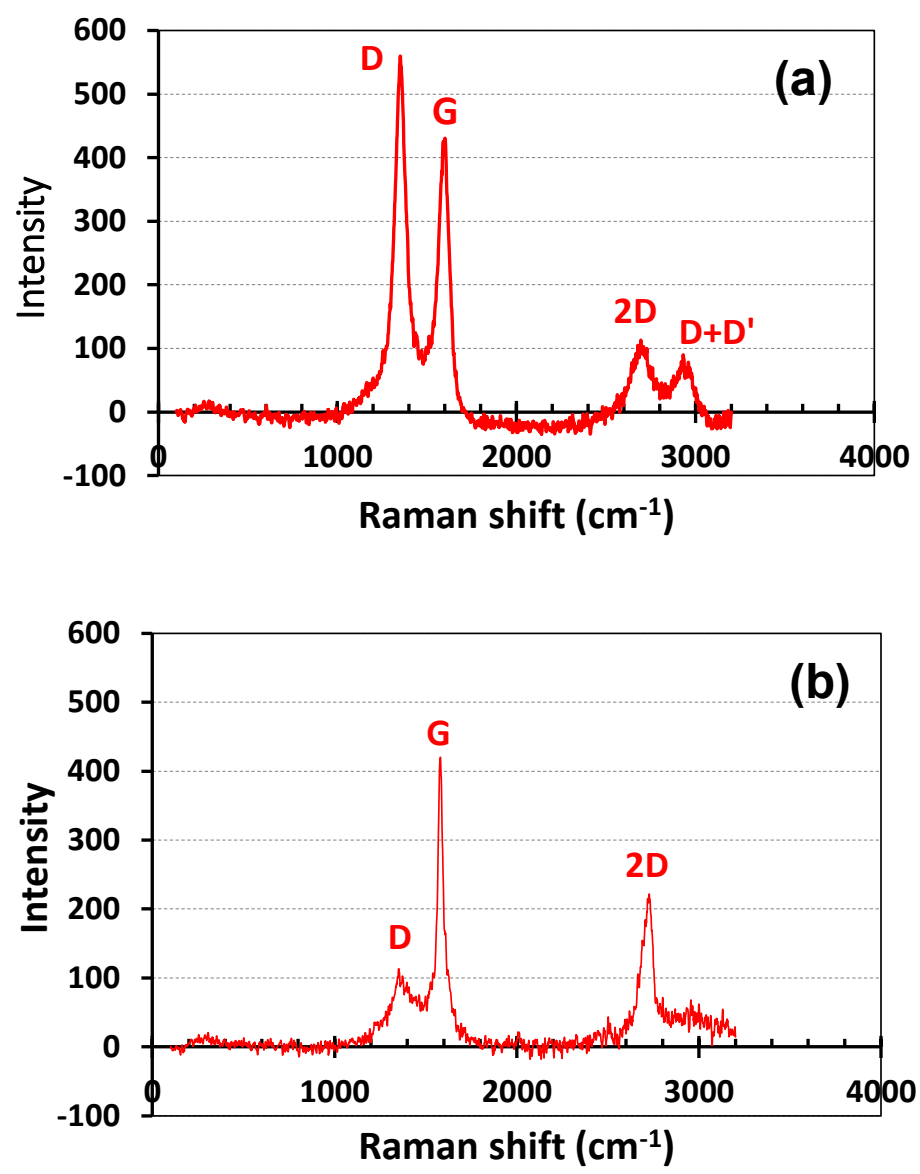

Figure 4. Raman spectra of (a) graphene I and (b) graphene II with a 514nm excitation laser wavelength. 


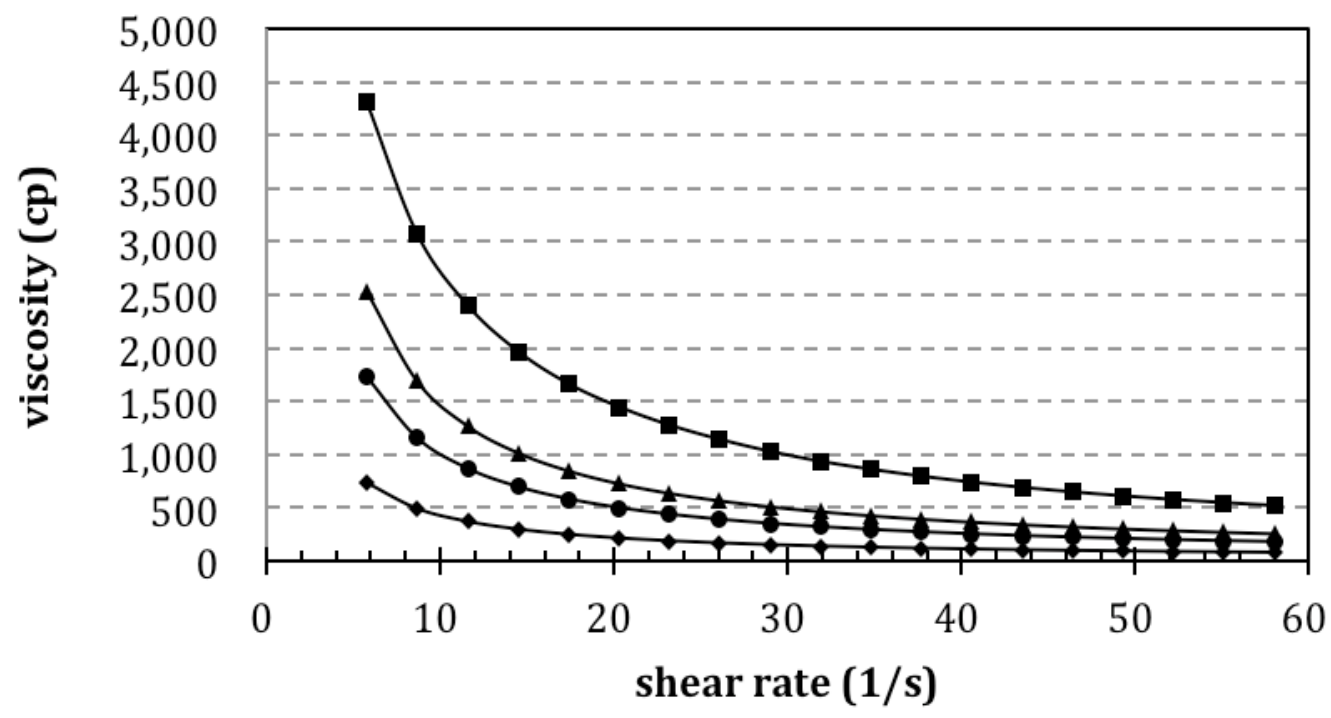

Figure 5. Viscosity as a function of the shear rate and sonicating time for non-aqueous slurries containing 2 wt. $\%$ of graphene I and 0.2 wt.\% CB. Sonicating time: $8 \min (\boldsymbol{\bullet}), 16 \min (\boldsymbol{\Delta}), 32 \min (\bullet)$, and $64 \min$ $(\diamond)$.

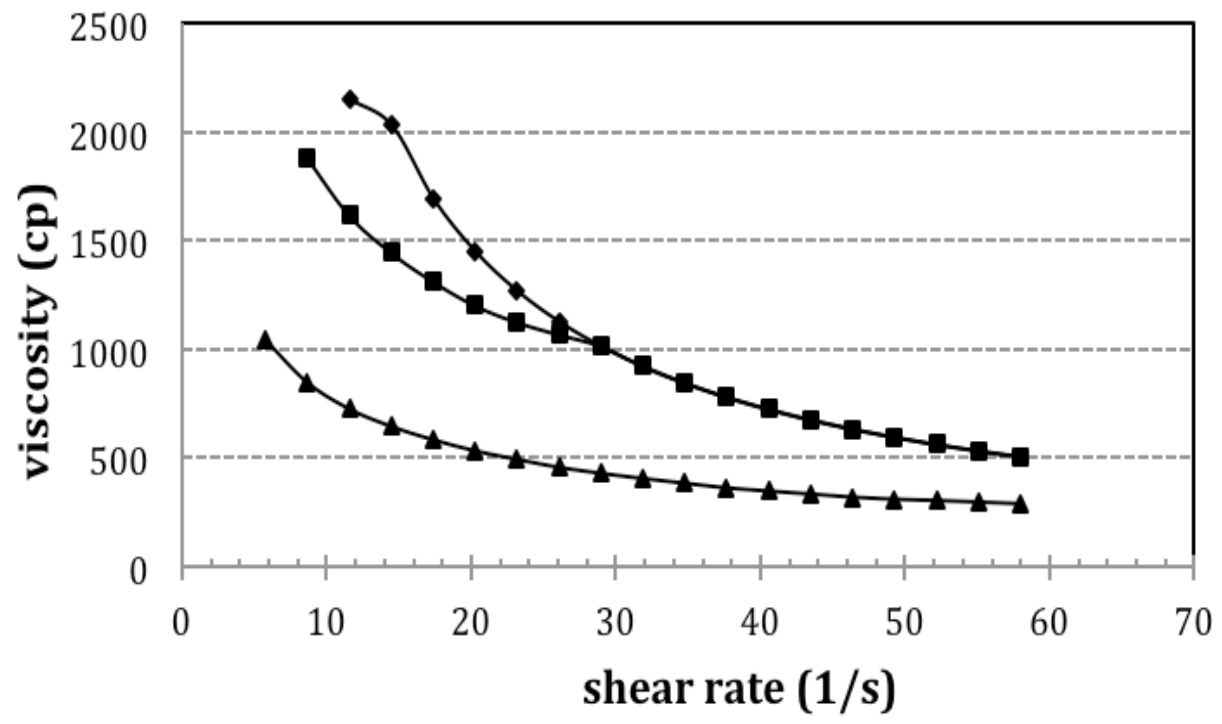

Figure 6. Viscosity as a function of the shear rate and solid loading for non-aqueous slurries. Solid loading

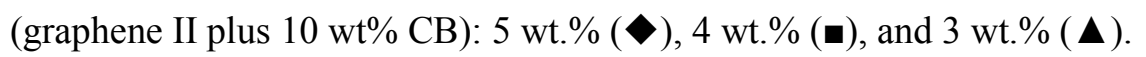




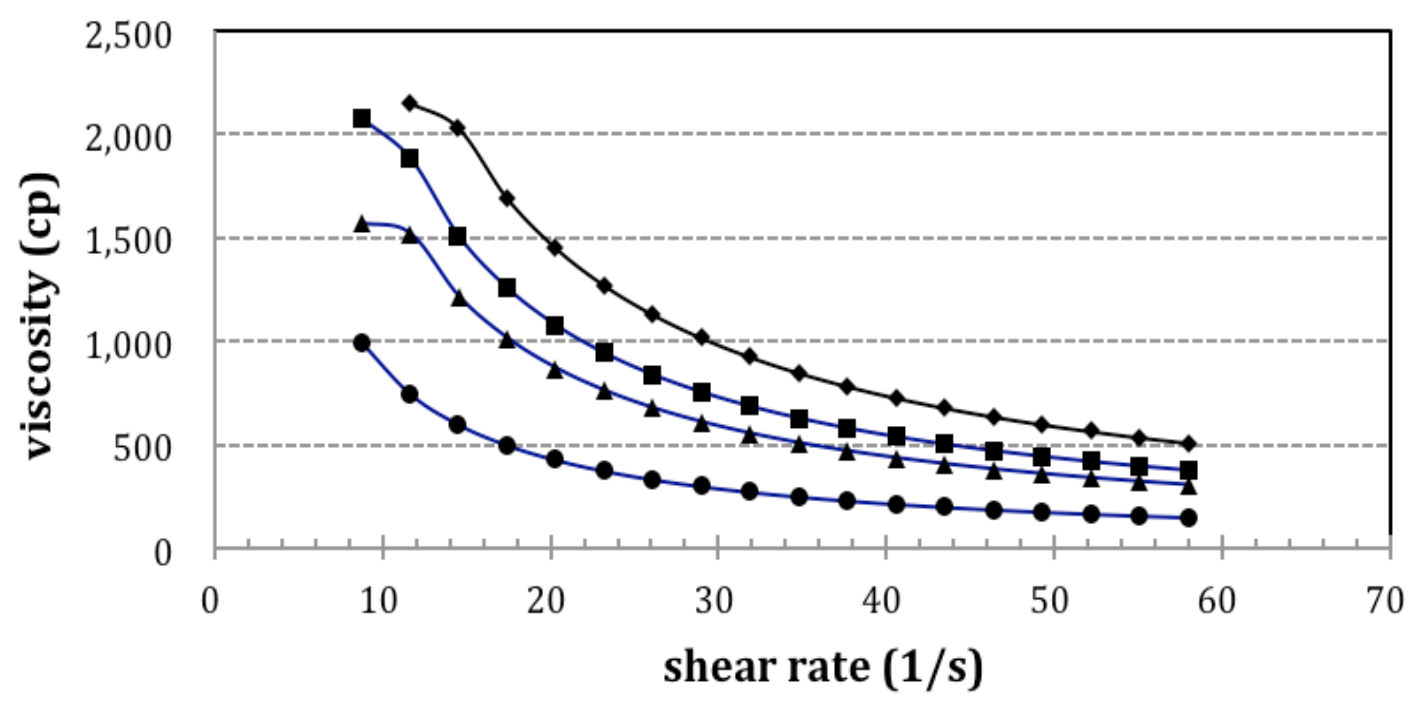

Figure 7. Viscosity as a function of the shear rate and solid loading for non-aqueous slurries. Solid loading

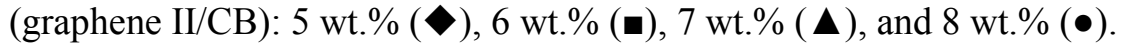

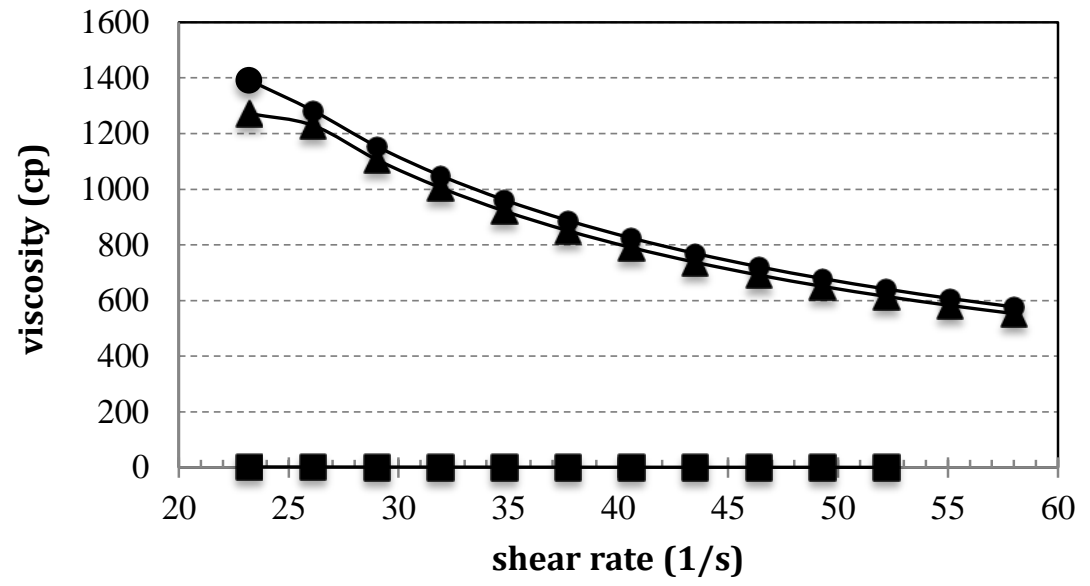

Figure 8. Viscosity as a function of the shear rate and solid loading for aqueous slurries with $0.015 \mathrm{M}$ SDS. Solid loading (graphene II/CB): 4 wt.\% (•) 5 wt.\% (•), and 6 wt.\% ( 


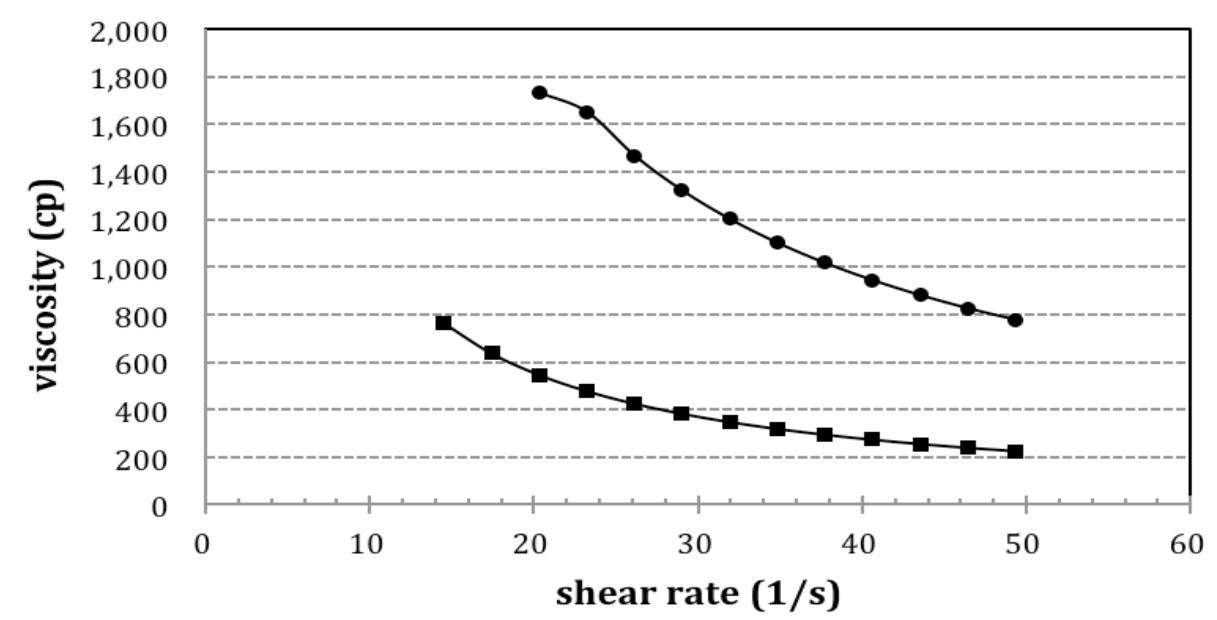

Figure 9. Viscosity as a function of the shear rate and solid loading for aqueous slurries with 0.03M SDS. Solid loading (graphene II/CB): 8 wt.\% (•) and 9 wt.\% (ロ).

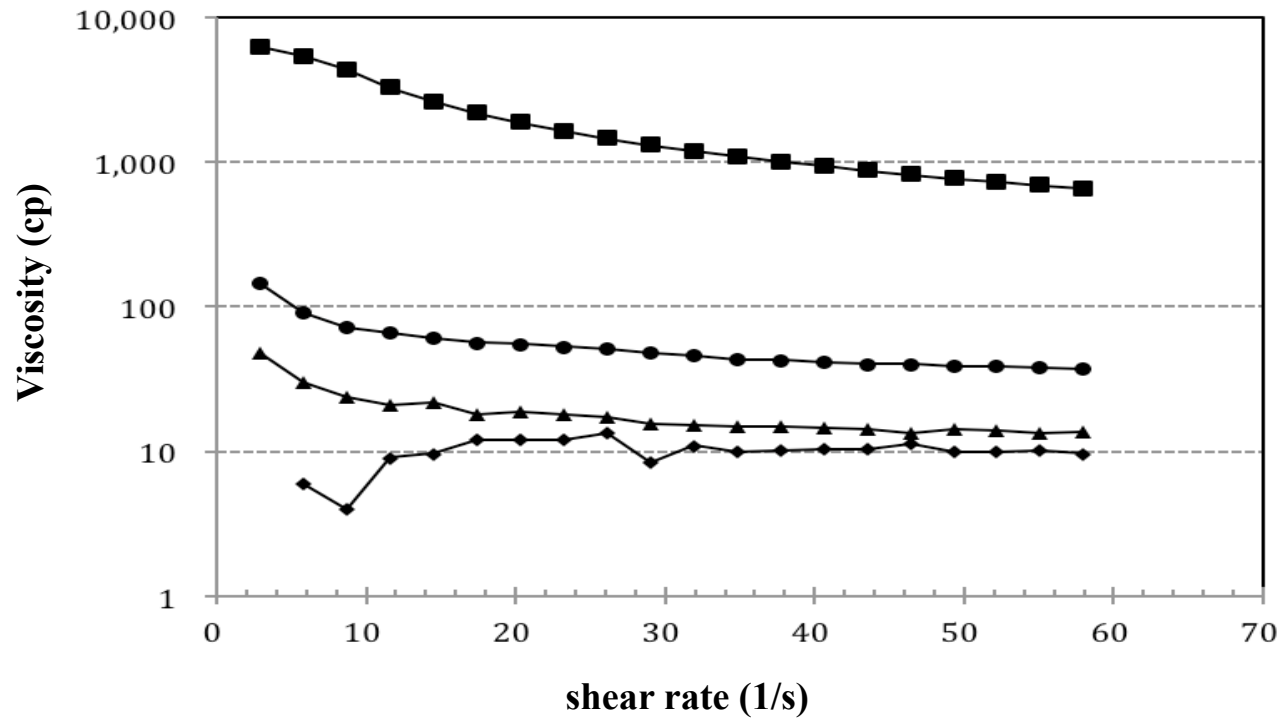

Figure 10. Viscosity as a function of the shear rate and solid loading for aqueous slurries with 0.06M SDS.

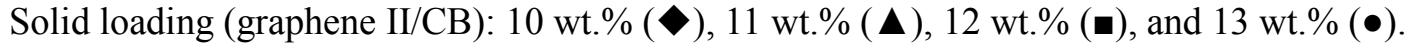




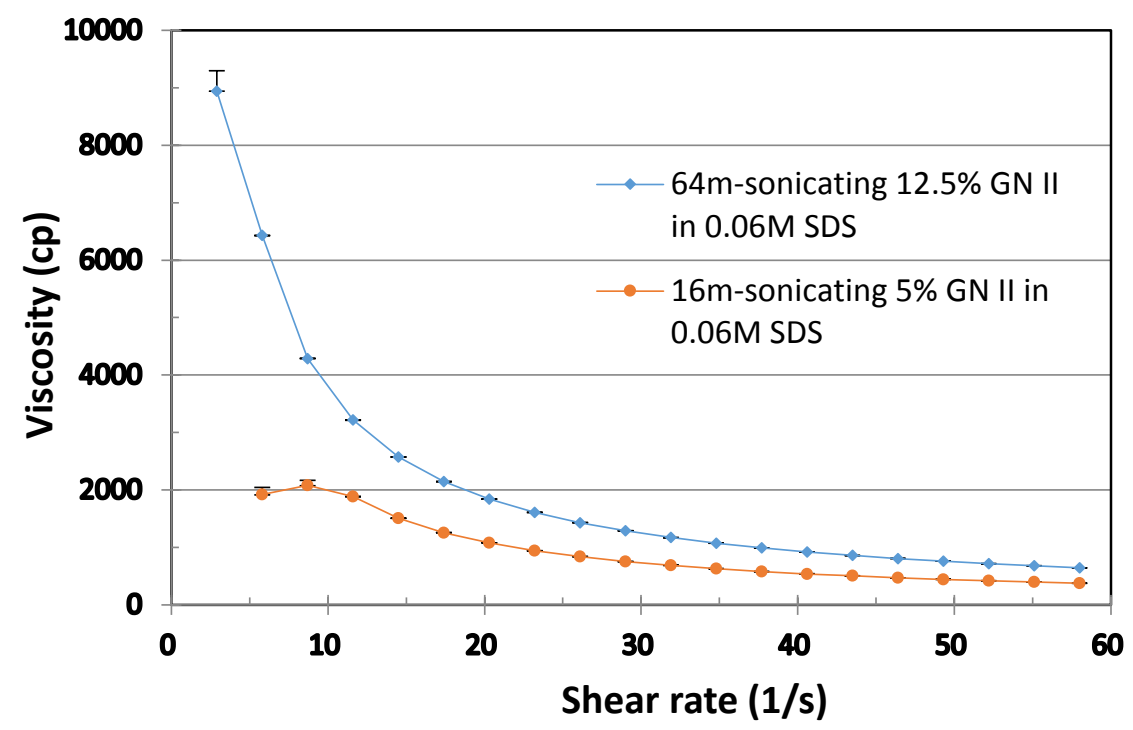

Figure 11. Viscosity as a function of the shear rate for aqueous slurries with $0.06 \mathrm{M}$ SDS but different sonicating times as indicated. Solid loading (graphene II/CB): 12.5 wt.\% for 64-minute sonication $(\diamond)$ and 5 wt.\% for 16-minute sonication $(\bullet)$. The error-bars in the figure represent the standard deviation and in many cases are smaller than the symbols shown in the figure, indicating the high reproducibility of the experiments.

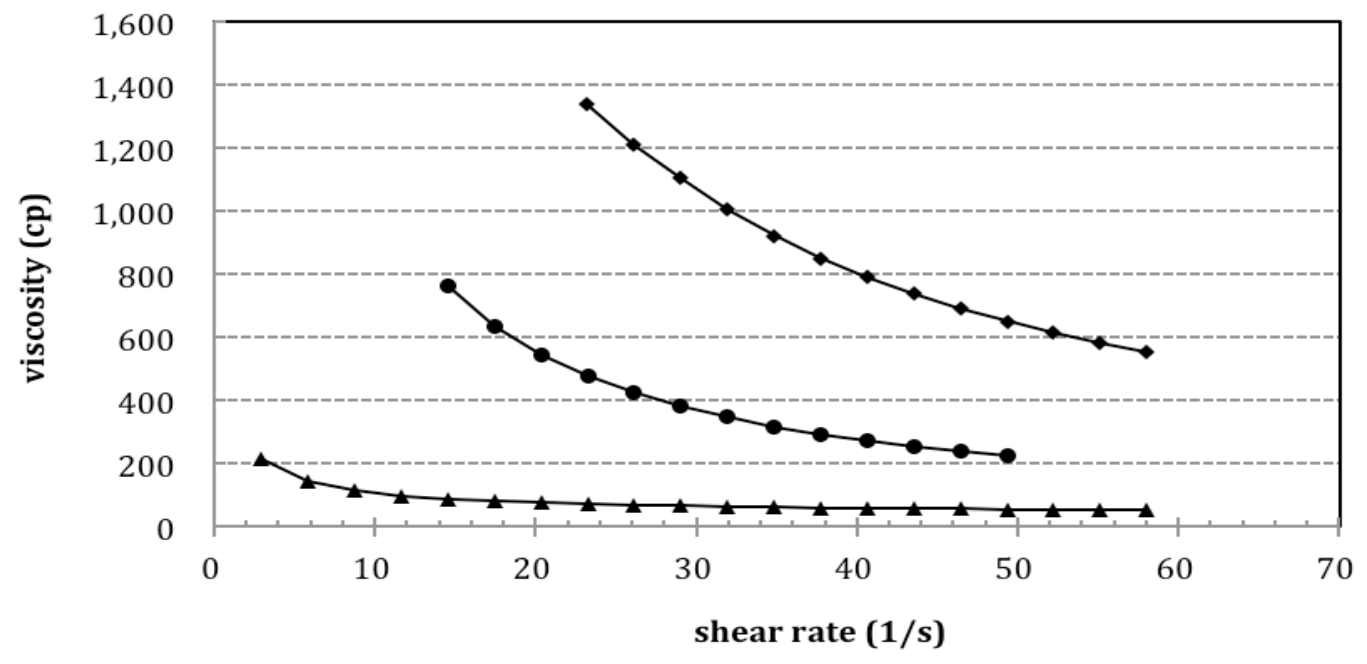

Figure 12. Viscosity as a function of the shear rate and SDS concentration for aqueous slurries. The graphene II/CB loading and SDS concentration: 6 wt.\% solid loading/0.015M SDS $(\diamond), 9$ wt.\% solid loading/0.03M SDS (•), and 13 wt.\% solid loading/0.06M SDS ( $\boldsymbol{\Delta})$. 

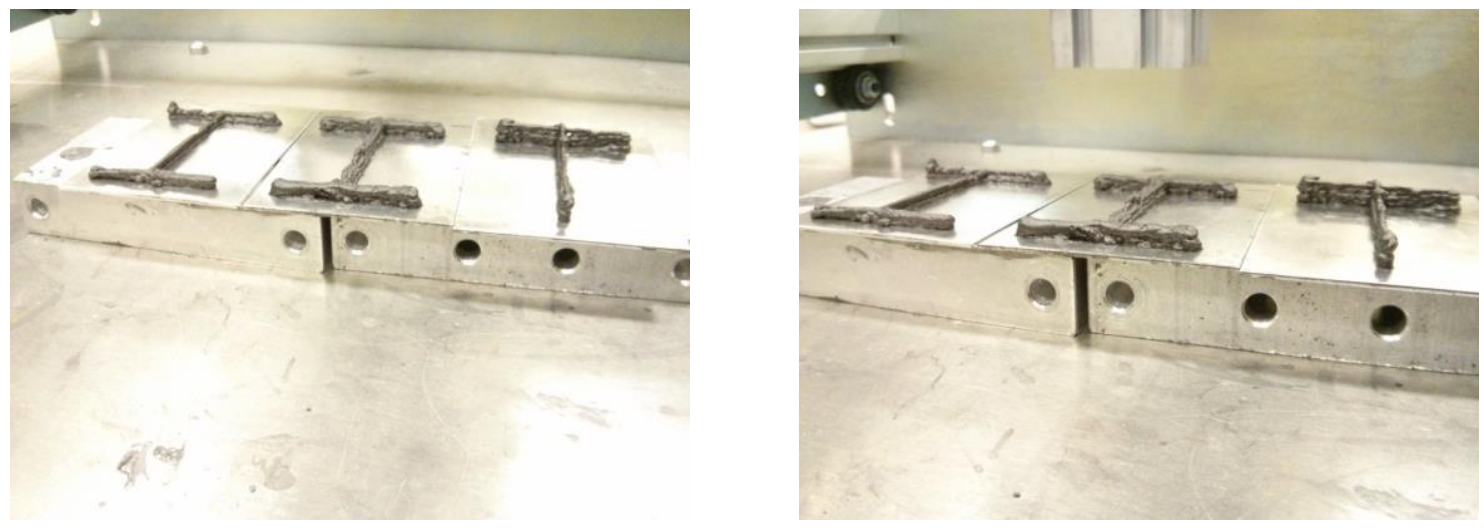

Figure 13. IIT letters fabricated through micro-extrusion of the aqueous slurry with 12.5 wt.\% graphene II/CB using an 18G nozzle.

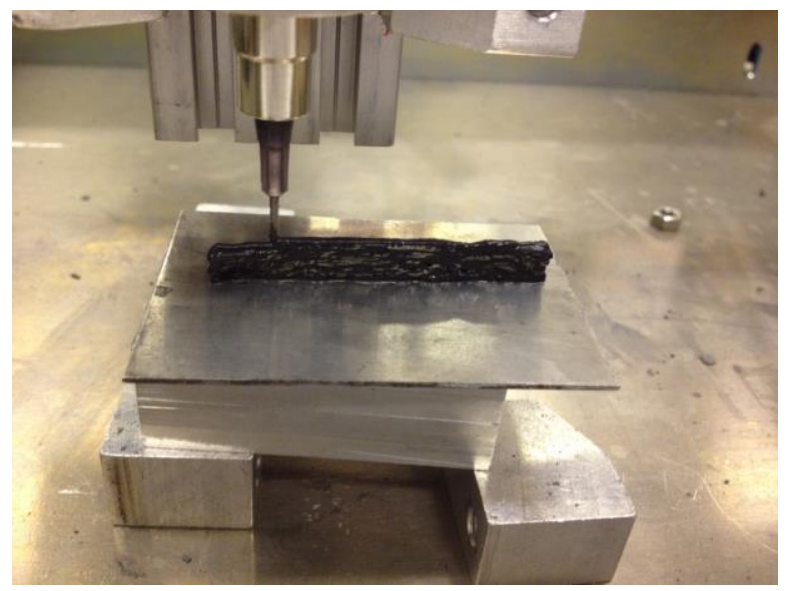

Figure 14. A single wall fabricated by stacking multiple lines from micro-extrusion of the aqueous slurry with 12.5 wt.\% graphene II/CB using an $18 \mathrm{G}$ nozzle. 